\title{
A left atrial hamartoma of mature cardiac myocytes
}

\section{Brief Report}

Cite this article: Rosen $\mathrm{K}$, Bishara JA, and Hakar M (2022) A left atrial hamartoma of mature cardiac myocytes. Cardiology in the Young 32: 1347-1349. doi: 10.1017/ S1047951121004972

Received: 21 July 2021

Revised: 2 November 2021

Accepted: 24 November 2021

First published online: 10 January 2022

\section{Keywords:}

Cardiac tumor; hamartoma; intracardiac mass; left atrial tumor; pediatric

\section{Author for correspondence:}

J. A. Bishara, MD, MBA, FAAP, Department of Pediatrics, Division of Pediatric Cardiology, Oregon Health and Science University, 707 SW Gaines St, Portland, OR 97239, USA.

Fax: 503-576-7970;

E-mail: bishara@ohsu.edu (c) The Author(s), 2022. Published by Cambridge University Press. This is an Open Access article, distributed under the terms of the Creative Commons Attribution licence (http:// creativecommons.org/licenses/by/4.0/), which permits unrestricted re-use, distribution and reproduction, provided the original article is properly cited.

\section{CAMBRIDGE} UNIVERSITY PRESS
Kate Rosen ${ }^{1}$, James A. Bishara ${ }^{2}$ (1) and Melanie Hakar ${ }^{3}$

${ }^{1}$ School of Medicine, Oregon Health and Science University, Portland, OR, USA; ${ }^{2}$ Department of Pediatrics, Division of Pediatric Cardiology, Oregon Health and Science University, Portland, OR, USA and ${ }^{3}$ Department of Pathology, Division of Anatomic Pathology, Oregon Health and Science University, Portland, OR, USA

\section{Abstract}

We present a case of a hamartoma of mature cardiac myocytes. This is an extremely rare tumour and the first reported paediatric case localised in the left atrium.

Primary cardiac tumours in children are rare. ${ }^{1}$ Although epidemiologic data are largely limited to institutional retrospective analysis, reports consistently identify that rhabdomyomas and teratomas account for the majority with prevalence of up to $90 \%$. Regardless of type, primary paediatric cardiac tumours are largely localised to the ventricles. ${ }^{1}$

Although often presenting asymptomatically or with only a murmur, these masses can be associated with significant morbidity and mortality including arrhythmias, outflow tract obstruction, valve dysfunction, and thromboembolic sequelae. ${ }^{2}$ As such, paediatric cardiac tumours are most often identified on a screening fetal echocardiogram or within a workup for some other clinical concern. ${ }^{2}$

Here, we describe the case of a 7-year-old boy found to have a left atrial mass that was pathologically identified as a hamartoma of mature cardiac myocytes. At the time of writing, there have been less than 30 reported cases of hamartoma of mature cardiac myocytes with few being paediatric and none localising to the left atrium. ${ }^{3}$

\section{Case}

A fully immunised 7-year-old boy with history of chronic normocytic anaemia presented to a local emergency department with acute systemic illness of fever, mild meningismus, bilateral conjunctivitis, diffuse rash, abdominal pain, loose stools, and dysuria for 7 days that was unchanged by 4 days of amoxicillin for workup of persistent fever with concerns for Kawasaki disease and multisystem inflammatory syndrome in children.

He was found to be non-toxic appearing with objective testing notable for an elevated temperature of 100.8 and elevated inflammatory markers. There was no focus of infection identified on the physical exam nor stigmata suggestive of endocarditis. A viral panel and COVID-19 polymerase chain reaction were negative, and blood cultures were obtained.

Out of concern for Kawasaki disease and multisystem inflammatory syndrome in children, a transthoracic echocardiogram was performed which revealed normal function, normal appearing coronary arteries, and a $10 \mathrm{~mm} \times 8 \mathrm{~mm}$ mass in the left atrium (Fig 1). The echocardiogram suggested the mass was attached to the atrial septum and may have had a connection to the anterior leaflet of the mitral valve. The mass had a non-homogenous appearance with an echo-lucency near the mitral valve. Using colour Doppler, flow could be seen within this echo-lucency although it was not clear if this was flow within the mass or into the mass from the left ventricle via the mitral attachment. There was no evidence of pulmonary venous or mitral valve obstruction. The echocardiographic appearance was suspicious for myxoma, endocarditis, and congenital cystic lesion of the mitral valve with or without associated endocarditis.

Complete blood count was notable for haemoglobin of 10.4 and mean corpuscular volume of 81.5 consistent with baseline. Erythrocyte sedimentation rate, C-reactive protein, and B-type natriuretic peptide were all elevated at initial presentations, but normalised over the course of his admission. Complete metabolic panel, urinalysis, and electrocardiogram were unremarkable. Blood cultures were negative for growth, and repeat viral testing including COVID-19 polymerase chain reaction were negative. His fever cleared with supportive care.

A gated CT of the heart was obtained to further delineate the mass. It revealed a non-homogenous left atrial mass with apparent attachment to the fossa ovalis suggestive of myxoma versus endocarditis (Fig 2). Other benign pathologies such as a haemangioma or atypical fibroma were considered. A CT of the head demonstrated no evidence of embolic events to the brain. Despite quality imaging, the atypical appearance of the lesion eluded diagnosis by non-invasive methods. Because of this, he was started on aspirin for platelet anti-aggregation and scheduled for resection. 


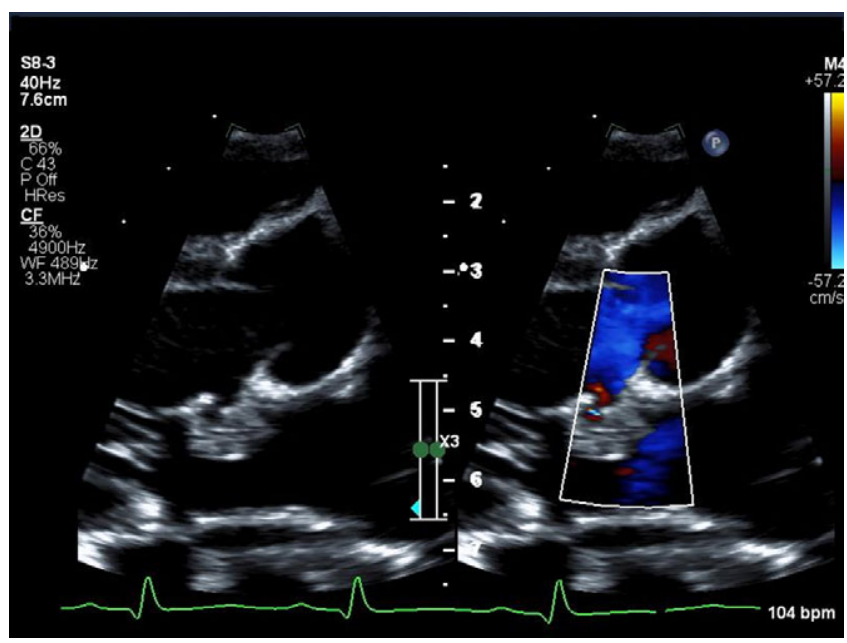

Figure 1. Long axis color compare focused on the mitral valve. A non-homogenous 8 $x 10 \mathrm{~mm}$ mass with appearent mitral valve attachment and color seen within the mass.

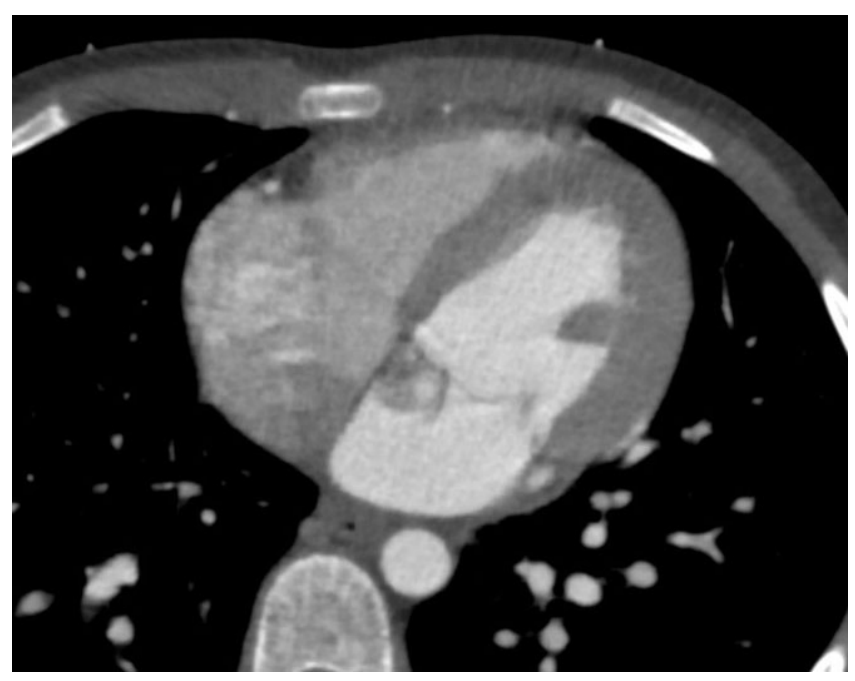

Figure 2. Gated axial CT with contrast shows a non-homogenous left atrial mass with attachment to the fossa ovalis and mitral valve. Some areas of the mass have a similar density to the contrast filling the left heart.

Resection was pursued primarily due to concern for atrial myxoma and partially treated endocarditis both of which would pose a risk of embolisation with a secondary reason of tissue diagnosis. Through a right atriotomy, the atrial septum was opened in a superior-inferior plan revealing the cardiac mass (Fig 3). During resection, the mass with a fibrous texture was found to be attached to the mitral valve and atrial septum. Removal from the mitral valve left, a significant defect in the anterior leaflet of the mitral valve which required repair with autologous pericardium.

The mass was a tan-white, nodular soft tissue fragment measuring 1.3 by 0.9 by $0.8 \mathrm{~cm}$. H \& E stains sections showed a haphazard proliferation of cardiac myocytes, blood vessels, and sparse adipose tissue without vacuolated cells and no myxoid changes (Fig 4). Of the three components described, the myocyte component was the most prominent and was consistent with differentiated mature striated cardiac myocytes.

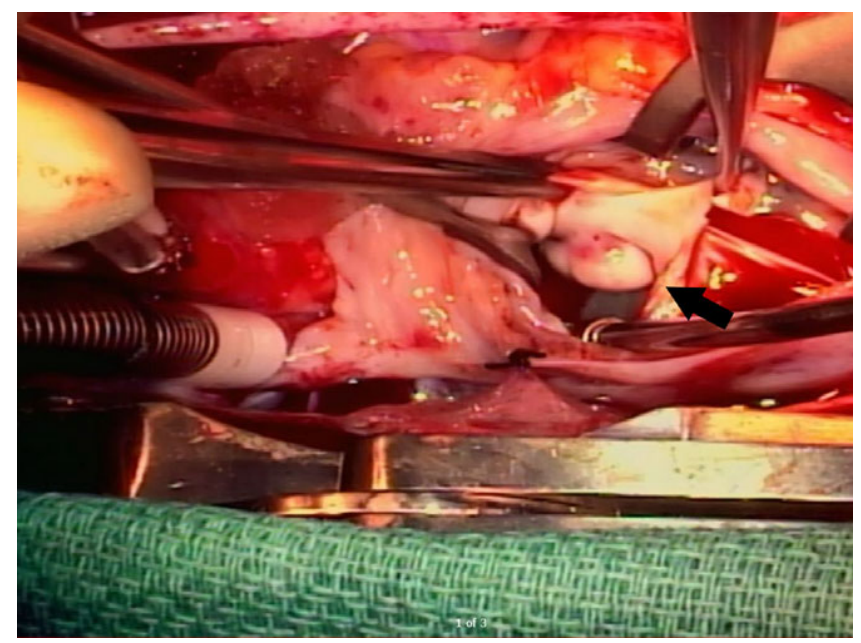

Figure 3. Intraoperative photograph of the tumor (arrow) in situ as viewed through a right atriotomy and opening of the atrial septum. The patient's head is oriented to the left of the image.

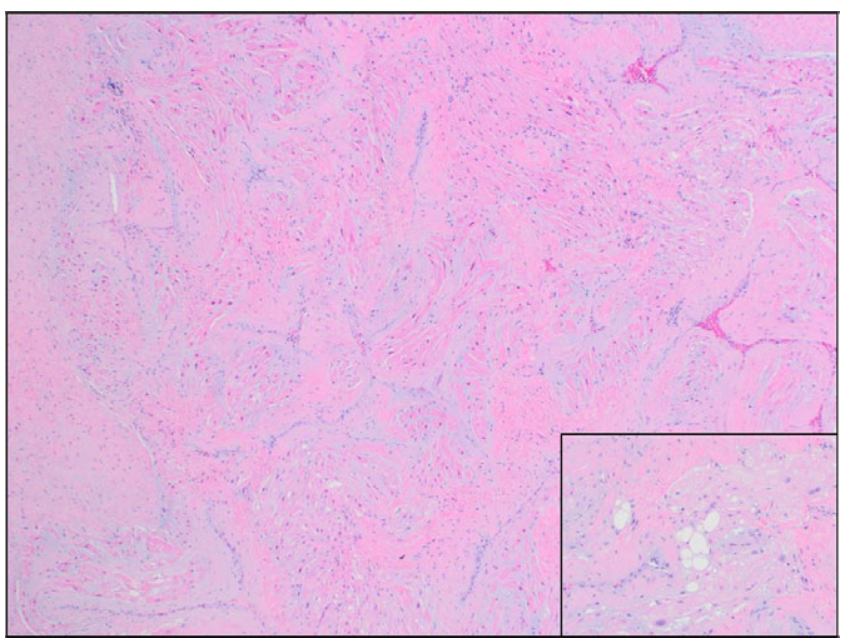

Figure 4. H\&E stained sections demonstrated a haphazard proliferation of cardiac myocytes and blood vessels (40x magnification). Inset: Focal arease of adipocytic differentiation were also present (100x magnification).

The operation and post-operative course were uneventful, and the patient recovered well. Post-operative transthoracic echocardiogram revealed no residual mass, normal function, and the new finding of mild mitral regurgitation with otherwise normal mitral valve function.

\section{Discussion}

This case highlights the clinical challenges faced when evaluating a newly identified intracardiac mass. When considering diagnostic imaging, echocardiography, CT, and MRI allow for precise description of the size and location of an intracardiac tumour, but have limited ability to differentiate between vegetation and types of cardiac tumours. ${ }^{4}$ The differential diagnosis for an intracardiac tumour of this size and shape includes fibroma, rhabdomyoma, angiosarcoma, and haemangioma. ${ }^{4}$ 
Of primary cardiac tumours, hamartomas of mature cardiac myocytes are rare with less than 30 cases reported in the literature and an average age of diagnosis in the twenties. ${ }^{5}$ The limited literature describes presenting symptoms on a spectrum from asymptomatic to ventricular arrhythmias to heart failure..$^{5-7}$ Given that our patient's initial echocardiogram was completed out of concern for cardiac involvement in a febrile state, concerns for an infectious mass were reasonable, but in retrospect, the mass was an incidental finding. In addition, following resolution of initial inflammatory symptoms, the patient demonstrated persistent fatigue, dyspnoea on exertion, chest pain, and palpitations which may be attributed to either the mass, sequelae of his acute illness, or some combination of both. The difficulty of making a definitive diagnosis through imaging for atypical cardiac masses may require resection for definitive diagnosis.

\section{Conclusion}

Primary cardiac tumours are rare in children. Cardiac hamartomas are the rarest of paediatric cardiac tumours, and this is the first report of a cardiac hamartoma in the left atrium with mitral valve involvement in a paediatric patient. Definitive diagnosis by imaging alone is difficult which necessitates resection of atypical masses for diagnosis.

Supplementary material. For supplementary material accompanying this paper visit https://doi.org/10.1017/S1047951121004972
Acknowledgements. Special thanks to Drs Ashok Muralidaran, Christian Fuss and Irving Shen for their contributions to this case.

Financial support. This research received no specific grant from any funding agency, commercial, or not-for-profit sectors.

\section{Conflicts of interest. None.}

\section{References}

1. Burke A, Virmani R. Pediatric heart tumors. Cardiovasc Pathol 2008; 17: 193-198.

2. Shi L, Wu L, Fang H, et al. Identification and clinical course of 166 pediatric cardiac tumors. Eur J Pediatr 2017; 176: 253-260.

3. Zhou X, Zhou Y, Zhaoshun Y, et al. Hamartoma of mature cardiomyocytes in right atrium: a case report and literature review. Medicine (Baltimore) 2019; 98: e16640.

4. Abuzaid AS, Gakhal M, Montgomery E, LaPoint R, Horn R, Banbury MK. Cardiac hamartoma: a diagnostic challenge. CASE (Philadelphia, Pa.) 2017; 1: 59-61. DOI 10.1016/j.case.2017.01.014.

5. Fealey ME, Edwards WD, Miller DV, Menon SC, Dearani JA. Hamartomas of mature cardiac myocytes: report of 7 new cases and review of literature. Hum Pathol 2008; 39: 1064-1071.

6. Dell'Amore A, Lanzanova G, Silenzi A, Lamarra M. Hamartoma of mature cardiac myocytes: case report and review of the literature. Heart Lung Circ 2011; 20: 336-340.

7. Dinh MH, Galvin JM, Aretz TH, Torchiana DF. Left ventricular hamartoma associated with ventricular tachycardia. Ann Thorac Surg 2001; 71: $1673-1675$. 